\title{
L'ENTREPRENEURIAT, LE TERRITOIRE ET LES CONDITIONS DE LEURS DYNAMIQUES CUMULATIVES
}

\author{
ENTREPRENEURSHIP, ITS TERRITORIAL CONTEXT \\ AND THE CONDITIONS OF CUMULATIVE DYNAMICS
}

\author{
Marcus DEJARDIN \\ Centre de Recherches sur l'Economie Wallonne \\ Facultés Universitaires Notre-Dame de la Paix \\ Rempart de la Vierge, 8, B-5000 Namur \\ Courriel : marcus.dejardin@fundp.ac.be
}

\begin{abstract}
Résumé
La contribution néo-autrichienne en matière d'entrepreneuriat est insérée dans un cadre d'analyse économique dynamique tenant compte du fait régional. Une introduction à la théorie kirznerienne de l'entrepreneuriat est d'abord proposée. Nous traitons plus spécialement de la notion de vigilance, de l'absence de coût entrepreneurial d'un point de vue analytique, ainsi que des coûts et des revenus attendus de l'activité entrepreneuriale relativement à d'autres activités en situation de système incomplet de marchés. Le choix occupationnel des individus et les effets de l'entrepreneuriat sur la croissance sont ensuite discutés dès lors que la dimension régionale des activités est introduite dans l'argumentation. Une présentation systémique des arguments du choix occupationnel et de la croissance conduit à mettre en évidence le fait que les effets de l'action entrepreneuriale sur la croissance peuvent difficilement être considérés indépendamment des effets en retour sur les paramètres de choix individuels, a fortiori en référence à un ensemble de ressources et d'activités économiques localisées en interrelation ou en interaction.
\end{abstract}

\begin{abstract}
Summary
The Neo-Austrian contribution as regards entrepreneurship is inserted within a dynamic setting taking account the regional fact. An introduction to the Kirznerian theory of entrepreneurship is first proposed. We deal more specifically with the concept of alertness, the absence of entrepreneurial cost from an analytical viewpoint, and the costs and incomes expected from entrepreneurial action, relative to other kinds of activities, in situation of incomplete system of markets. The occupational choice by individuals and the effects of entrepreneurship on economic growth are then discussed since the regional dimension of economic activities is introduced into the argumentation. A systemic approach of occupational choice and growth results in highlighting that the effects of entrepreneurial action on economic growth cannot be considered without difficulty independently of the feed-back effects on the individual occupational choice parameters, a fortiori in reference to a whole set of localized economic resources and activities in interrelationship or interaction.
\end{abstract}

\section{Remerciements}

L'auteur remercie de leurs suggestions et commentaires Vinciane Dermien, Michel Mignolet, Pierre Perrin ainsi que les participants à la session «Perspectives autrichiennes en économie régionale » organisée à l'occasion du XLème colloque de l'ASRDLF, Bruxelles, 1-3 septembre 2004.

Mots-clés : Entrepreneuriat, territoire, croissance, Kirzner. Key-words: Entrepreneurship, territory, growth, Kirzner.

Classification JEL : B53, M13, O12, R11. 


\section{INTRODUCTION}

A la suite des travaux pionniers de KIRZNER (1973), les économistes néo-autrichiens se sont interrogés sur la contribution dynamique de l'entrepreneur. Dans ce cas, il s'agit de comprendre les processus par lesquels l'entrepreneur contribuerait, non pas seulement à la découverte du stock d'opportunités de profit, éventuellement alimenté par le changement d'origine exogène des conditions économiques, mais encore à la création de nouvelles opportunités de profit. Par son action, l'entrepreneur entrerait alors dans une explication de phénomènes cumulatifs et autoentretenus. Ainsi, l'exploitation des opportunités de profit en créerait de nouvelles qui motiveraient leur recherche et leur exploitation par l'entrepreneur, ce qui en conséquence aboutirait à de nouvelles opportunités et ainsi de suite (HOLCOMBE, 1998).

Dans cette contribution, nous insérons l'approche néo-autrichienne en matière d'entrepreneuriat dans un cadre d'analyse économique dynamique tenant compte du fait régional. Dans ce contexte, les choix occupationnels des individus en particulier ne peuvent être envisagés indépendamment de leurs effets agrégés en termes de revenus attendus (DEJARDIN, 2000a, 2004). Nous tâchons d'identifier les résultats d'une fertilisation croisée. En quoi la prise en compte de la dimension régionale permet-elle de préciser les conditions des processus cumulatifs qui seraient à l'œuvre dans le processus entrepreneurial néo-autrichien ? Inversement, dans quelle mesure l'apport néo-autrichien ouvre-t-il de nouvelles perspectives pour l'appréciation du résultat des choix occupationnels des individus dans le contexte régional ?

La contribution traite plus spécifiquement des décisions des agents économiques au travers de l'analyse du développement de l'offre de biens et services, des revenus attendus et des effets en retour sur les décisions. Le contexte institutionnel des décisions n’est quasiment pas abordé. En outre, les traits psychologiques des agents et leur subjectivité sont tenus pour donnés ${ }^{1}$.

Pour ce qui concerne les références néo-autrichiennes, précisons que la problématique de l'entrepreneuriat est exclusivement examinée à partir de la théorie de la «découverte entrepreneuriale équilibrante » et des développements qu'ont pu lui apporter les épigones de KIRZNER. La dimension régionale de l'analyse se limite à intégrer les effets attendus de l'organisation spatiale des activités et, en particulier, de la proximité sur un ensemble de ressources et d'activités économiques en interrelation ou en interaction.

L'article s'articule de la manière suivante. Dans la première section, une introduction à la théorie kirznerienne de l'entrepreneuriat est proposée. Nous traitons plus spécialement de la notion de vigilance, de l'absence de coût entrepreneurial d'un point de vue analytique, ainsi que des coûts et des revenus attendus de l'activité entrepreneuriale relativement à d'autres activités en situation de système incomplet de marchés. Les effets de l'entrepreneuriat sur la croissance sont aussi examinés. Dans la deuxième section, le choix occupationnel des individus et les effets de l'entrepreneuriat sur la croissance sont discutés dès lors que la dimension régionale des activités est introduite dans l'argumentation. La troisième section présente les résultats d'une réflexion sur les conditions pour une dynamique entrepreneuriale et territoriale cumulative compte tenu des arguments et du cadre d’analyse précédemment avancés. 


\section{L'ENTREPRENEUR KIRZNERIEN MIS EN PERSPECTIVE}

Le processus qui conduit le marché à tendre vers l'équilibre est au coeur des préoccupations de recherche d'économistes néo-autrichiens dont Israel KIRZNER est sans aucun doute l'un des théoriciens pionniers ${ }^{2}$. Dans ce processus, la connaissance et la découverte entrepreneuriale jouent un rôle fondamental. Cela étant, la contribution théorique kirznerienne se nourrit d'une synthèse d'arguments tirés des œuvres de HAYEK et de MISES.

KIRZNER attribue de manière analytique une fonction économique majeure et tout à fait spécifique à l'entrepreneur. Par son action, motivée par la réalisation d'un profit, l'entrepreneur contribue à générer de la connaissance partagée par les acteurs économiques et de ce fait, réduit leur ignorance. De la sorte, il agit positivement sur la coordination des plans de demande et d'offre sur le marché (KIRZNER, 1997). Le profit entrepreneurial est un profit pur qui n'est à mettre en rapport avec l'utilisation d'aucun facteur de production quel qu'il soit. Il résulte d'une décision simultanée d'achat et de vente à la suite de la découverte de différences de prix avantageuses dont l'existence est fondée sur l'ignorance des agents quant à l'offre et la demande exactes. Selon KIRZNER (1973), la découverte d'opportunités de profit jusque-là ignorées des agents économiques ne nécessiterait donc aucun investissement particulier.

Le processus de production n'est pas exclu des conditions de réalisation d'un profit entrepreneurial. Dans ce cas, l'entrepreneur découvre le bénéfice qu'il pourrait tirer, compte tenu des prix, de l'utilisation de facteurs de production pour la transformation de matières et pour leur commercialisation ou pour la prestation de services. La question du délai entre l'achat des intrants et la vente finale n'importe pas. Ce qui compte, en revanche, c'est la décision ex ante qui aura été prise lorsque l'opportunité de profit résultant d'une appréciation du processus de production dans sa globalité aura été découverte.

A l'origine des opportunités de profit, il y a l'ignorance des agents économiques quant à leurs plans croisés d'achat et de vente. De l'ignorance découle un stock d'opportunités de profit que l'action entrepreneuriale va réduire. C'est la fonction équilibrante de l'entrepreneur dans le processus de marché. Sans ignorance, il ne peut y avoir d'opportunités de profit. L'action entrepreneuriale est, éventuellement, suivie de l'action mécanique des imitateurs. Les forces du marché tendent vers l’équilibre compétitif.

Le stock d'opportunités de profit est alimenté de manière exogène par le changement des conditions économiques qui peuvent être déterminées par de nombreux facteurs nonentrepreneuriaux. Le progrès technique, quand il résulte de la décision délibérée de Recherche et Développement, est considéré comme exogène à l’action entrepreneuriale.

A l'origine de la découverte d'opportunités de profit, se trouve la manifestation de la vigilance (alertness), le trait caractéristique de l'entrepreneur kirznerien. Cette vigilance n'est pas une affaire de connaissance préalable. D'une part, il n'est pas possible de connaître ce que l'on ignore. D'autre part, si l'entrepreneuriat se résumait à exploiter sa connaissance du marché, alors il n'y aurait plus de raison solide de distinguer l'entrepreneuriat des facteurs de production, et le dégagement d'un profit pur plutôt que la rémunération d'un service. La vigilance signifie davantage savoir où chercher des données relatives au marché que la connaissance de ces données (KIRZNER, 1973, p. 67). Si les agents avaient la connaissance des opportunités de 
profit, ils pourraient les saisir à la manière des gestionnaires de firme qui maximisent une fonction de profit selon une technologie, des dotations et des paramètres connus et reçoivent d'ailleurs à cette fin (et pour la location de leurs connaissances) une rémunération de la part des propriétaires. Il s'agit pour les gestionnaires d'exécuter un programme ; il n’y a ni découverte, ni décision ou profit entrepreneurial dans ce cas.

Au vu des arguments qui précèdent, DEMSETZ n’hésite pas à avancer l'idée qu'en fin de compte, ce qui serait l'essence de l'entrepreneuriat kirznerien, exprimé en termes plus familiers, serait la chance (luck). En effet, note DEMSETZ (1983, p. 277-279), la vigilance est tout ce qui importe. Le profit ne résulte pas d'une intention délibérée en fonction de laquelle l'entrepreneur aura consacré du temps et de l'énergie, ou d'autres ressources. Et l'auteur de prendre ses distances en affirmant que, s’il admet que la vigilance vis-à-vis des opportunités de profit peut être exercée à un coût quasi nul, l'absence totale de coût est difficilement concevable. La vigilance détourne l'esprit de l'exercice d'autres tâches. Et il y a dès lors bel et bien un coût attaché à la vigilance. En outre, quelle peut être la contribution de l'entrepreneuriat au progrès si celui-ci ne développe son action qu'à partir de quelque situation chanceuse ? Cela étant, il convient sans doute, tout d'abord, de rappeler la préoccupation analytique de KIRZNER lorsque celui-ci met en évidence, dans le processus de marché, la fonction équilibrante spécifique de l'entrepreneur et la rétribution de celui-ci au travers du profit pur. La distinction analytique n’empêche pas de reconnaître qu'en réalité, un même agent économique puisse remplir d'autres fonctions (l'exécution mécanique d'un plan de production, la charge de risque ou d'assurance, la charge d'incertitude,...) compte tenu de ses qualités ou de ses actifs pris au sens large. Ceux-ci doivent alors être considérés comme des facteurs de production pour la location desquels le marché (dans l'ignorance plus ou moins corrigée par l'action de l'entrepreneuriat) fixera la rémunération. On note ensuite très sommairement que KIRZNER (1979 ; pp. 154-181 et pp. 258259) reconnaît lui-même le rôle joué par la chance. Il insiste toutefois sur le fait que la manifestation de cette dernière ne peut être le seul élément dont découle le profit entrepreneurial. Si tel était le cas, il faudrait alors parler de gains tombés du ciel (windfall gains). L'élément indissociable de la chance est ici constitué par l'action humaine à la MISES. Sans celle-ci, il ne peut y avoir de profit entrepreneurial.

Les commentaires de DEMSETZ en appellent d'autres qui nous conduiront enfin à considérer l'apport d'une analyse statique, puis dynamique, tenant compte du fait régional. Nous nous attacherons à une brève discussion du profit entrepreneurial en regard du coût d'opportunité de la vigilance ainsi qu'à la contribution attendue de l'action de l'entrepreneur kirznerien en termes de croissance économique.

\subsection{Profit et coût entrepreneuriaux}

La vigilance entrepreneuriale n'entraîne aucun investissement. Elle est sans coût. KIRZNER insiste sur ce point ${ }^{3}$. Il lui permet de fonder analytiquement une fonction spécifique à l'entrepreneur. La perception d'une opportunité de profit est ou n'est pas dans le chef de l'entrepreneur. Elle n'est pas le fruit d'une recherche délibérée. Elle implique la surprise (HARPER, 2003, p. 28, citant KIRZNER, 2000, p. 18). L'entrepreneur ne décide pas de consacrer de son temps ou d'autres moyens à la vigilance. De ce point de vue, le coût de la fonction spécifique de l'entrepreneur apparaît nul. 
Notons que, lorsque KIRZNER distingue, à partir du critère de (non-)déployabilité, la vigilance d'autres ressources utiles dans la prise de décision, telles que le temps, on ne peut conclure que la vigilance et la perception du profit soient instantanées sans devoir émettre cette hypothèse supplémentaire. La non-déployabilité de la vigilance n’implique pas que la vigilance soit atemporelle. Un laps de temps peut s'écouler pour le soupçon ou la perception. Il doit alors être déduit du temps disponible pour la prise de décision. Evénement non délibéré, le soupçon de profit peut être immédiatement écarté ou, en revanche, accepté par l'entrepreneur. Dans ce cas, il représente un coût d'opportunité identifiable par l'entrepreneur.

Ajoutons que l'attitude de vigilance elle-même peut être consommatrice de temps. En outre, la découverte d'une opportunité de profit requiert une appréciation a priori, subjective, révélatrice du potentiel de l'opportunité et de sa profitabilité. L'exercice d'évaluation est un processus qui nécessite que l'entrepreneur y consacre son attention pour un laps de temps plus ou moins long. KIRZNER écarte la difficulté en préférant parler de perception plutôt que d'appréciation ou d'évaluation, termes qui impliquent la recherche d'une fin et le détournement de ressources pour y parvenir. La question mérite sans doute un débat. Néanmoins, admettons la préoccupation analytique de KIRZNER et l'absence de coût lié à la vigilance.

La réalisation d'une opportunité de profit peut être coûteuse en temps (KIRZNER, 1973, p. 48). Mais la réalisation doit définitivement être distinguée de la découverte. Dès lors qu'est connue l'opportunité de profit par l'entrepreneur, celui-ci peut en confier la réalisation au gestionnaire (moyennant la rémunération de son travail et des différents intrants nécessaires, le profit pur revenant à l'entrepreneur). Cette proposition ne tient qu'à l'hypothèse selon laquelle l'entrepreneur puisse trouver un gestionnaire et, ce dernier, toutes les ressources nécessaires sur les marchés. L'incomplétude du système de marchés peut expliquer que la fonction spécifique de l'entrepreneur doive être associée à d'autres fonctions économiques pour l'accomplissement desquelles l'investissement de ressources s'avère nécessaire. En pratique, il apparaît que l'incomplétude du système de marchés soit plutôt la règle. Il n'existerait pas d'entrepreneurs purs, n'assumant que la fonction entrepreneuriale. En revanche, un même agent assumerait différentes fonctions. C'est ainsi qu'un même agent économique puisse être l'entrepreneur, le bailleur de fonds et l'assureur supportant une activité économique. Cette situation est d'autant plus probable si, comme HARPER (2003, p. 27) le précise, la vigilance se réfère à une information de nature tacite, dont la prise de conscience par l'intéressé est malaisée et qui est aussi difficilement communicable à autrui.

En théorie si l'on pose l'incomplétude du système de marchés, ou en pratique, l'entrepreneur ne peut donc réaliser le profit entrepreneurial pur sans assumer lui-même une série de fonctions extra-entrepreneuriales. Il n'est pas possible d'être entrepreneur et de réaliser son profit séparément d'autres activités productives.

Cela étant, l'entrepreneuriat est une fonction qui n'est pas l'apanage d'une classe particulière d'individus. Tout un chacun peut être entrepreneur. Et il apparaît dès lors que le temps et les autres ressources consacrés à l'assortiment de l'acte entrepreneurial et des autres activités nécessaires à la réalisation du profit qui n’ont pu être confiées à autrui via un marché ne peuvent être investis dans d'autres activités non entrepreneuriales éventuellement rémunératrices. Le temps étant une ressource limitée qui peut connaître des usages alternatifs, il devient l'objet d'un 
problème d'allocation optimale (BECKER, 1965) ${ }^{4}$. Il en va ainsi de toutes les autres ressources économiques.

Le problème posé se résume à un choix occupationnel : d'une part, consacrer son temps, ses capacités et des ressources dans un projet faisant suite à la découverte d’opportunités de profit, contre un profit pur et la rémunération des facteurs de production nécessaires à la réalisation de l’opportunité de profit; d'autre part, offrir son temps et ses capacités contre une rémunération fixée sur le marché du facteur de production ad hoc. La situation d'entrepreneur qui comprend l'acte entrepreneurial s'oppose à celle de facteur de production salarié exécutant mécaniquement des tâches délibérées. Le résultat de la comparaison des revenus relatifs attendus et le choix du maximum expliquent la sélection de l'une ou l'autre de ces occupations.

\subsection{Entrepreneuriat et croissance économique}

L'articulation de l'entrepreneuriat kirznerien et de la croissance économique emprunte de manière schématique différents canaux rendus progressivement explicites dans la littérature au rythme de son développement.

Un premier canal est défini par l'effet favorable de l'action entrepreneuriale sur la coordination des plans des acteurs économiques sur le marché. En arbitrant les propositions d'achat et de vente lorsque celles-ci offrent une opportunité de profit, l'entrepreneur kirznerien améliore l'efficience de marché. Ce faisant, l'économie devient plus compétitive au sens où elle permet une plus grande satisfaction des consommateurs. Le raisonnement vaut aussi en matière d'activités productives, lorsque l'entrepreneur évalue l'écart entre les prix des facteurs de production pour leur utilisation à des fins de productions alternatives, voire pour la même production. Sa contribution à la correction de l'ignorance ou de l'erreur des agents économiques peut donc avoir pour origine la découverte d'opportunités de profit par comparaison de situations prévalant sur différents marchés (HARPER, 2003, pp. 22-23).

L'incorporation du progrès technique dans le modèle offre la possibilité de nouvelles opportunités de profit sans pour autant remettre fondamentalement en cause la fonction spécifique de l'entrepreneur kirznerien. Il importe seulement d'accepter l'idée que la connaissance de l'opportunité de profit ne peut exister préalablement à la découverte entrepreneuriale et que, dès lors que la connaissance sera partagée, l'effet de la concurrence conduira inexorablement le profit à néant (KIRZNER, 1985). SCHUMPETER (1911) attache à l'entrepreneur une fonction d'innovation qui tire l'économie de son état routinier, de sorte que l'entrepreneur schumpeterien apparaît assumer une fonction déséquilibrante. L'entrepreneur schumpeterien est créateur d'opportunités de profit, là où l'entrepreneur kirznerien les découvre. KIRZNER (1999, pp. 13-15) montre comment la fonction équilibrante qu'il développe et l'approche schumpeterienne sont bel et bien réconciliables. L'innovation est perturbante. Et KIRZNER de citer l'exemple des effets de l'arrivée de l'automobile sur l'industrie de l'attelage de chevaux. Cependant, que l'on songe à l'utilisation des ressources dans cette industrie alors que le progrès technologique permettait la création d'un engin autopropulsé. L’opportunité de profit dans la création d’une industrie automobile ayant été vérifiée par la suite vu les résultats engrangés, force est de reconnaître alors la situation de déséquilibre ou un déficit de coordination que les entrepreneurs pionniers de l'automobile ont en fait permis de réduire ${ }^{5}$. 
En résumé, il ressort que l'entrepreneur kirznerien, d’une part, en découvrant par l'arbitrage des opportunités de profit précédemment inexploitées contribue à augmenter l'efficience économique et technologique de l'économie et, d'autre part, en découvrant les possibilités offertes par le progrès technologique qui permet aussi une utilisation plus efficiente des ressources, agit positivement sur la productivité des facteurs. Ainsi, l'action de l'entrepreneur apparaît au coeur d'une explication de la croissance économique (BOETTKE et COYNE, 2003, pp. 73-74).

HOLCOMBE (1998, 2003) endogénéise les opportunités de profit en les faisant découler de l'action entrepreneuriale ${ }^{6}$. La concrétisation des opportunités de profit par les entrepreneurs se traduit par un accroissement des actifs matériels et immatériels. Ces créations d'actifs, notamment lorsqu'elles prennent la forme de connaissances nouvelles, peuvent indirectement contribuer à la croissance par un effet de débordement (ROMER, 1986, 1990). Dans le cadre explicatif avancé par KIRZNER, elles donnent avant tout l'occasion de nouvelles combinaisons à découvrir. HOLCOMBE (1998, 2003) d'évoquer les nouvelles opportunités de profit rendues possibles par la réalisation des opportunités de profit découvertes par Xerox dans l'industrie de la reprographie et leur application dans l'industrie des ordinateurs et des systèmes d'exploitation. Xerox fut incapable de capitaliser sur ses produits et ce fut Macintosh et ensuite Microsoft qui découvrirent les opportunités de profit à saisir dans l’informatique.

Dans la section suivante, le choix occupationnel des individus et les effets à attendre de l'entrepreneuriat sur la croissance sont discutés dès lors que la dimension régionale des activités est prise en compte.

\section{L'INSCRIPTION TERRITORIALE DE L'ENTREPRENEURIAT KIRZNERIEN}

Jusqu’à la précédente section, les choix occupationnels des agents économiques ainsi que les effets attendus de l'entrepreneuriat sur la croissance économique ont été envisagés indépendamment de leur dimension régionale. Or la dimension régionale ne serait pas sans conséquence sur les uns et les autres. Cette section se propose d'expliciter les arguments en présence et leur articulation.

\subsection{Le facteur régional dans le choix occupationnel}

L'assortiment de l'acte entrepreneurial et des autres activités nécessaires à la réalisation du profit pur qui n'ont pu être confiées à autrui via un marché découle entre autres choses d'un choix occupationnel posé par l'individu, arbitrant entre différentes situations professionnelles (dont la situation d'entrepreneur) caractérisées chacune par une espérance de revenus ${ }^{7}$.

Plusieurs modèles de choix occupationnels incluant la situation d'entrepreneur ont été proposés dans la littérature depuis les travaux pionniers de Frank KNIGHT (1921). Citons notamment LUCAS (1978), KANBUR (1979), KIHLSTROM et LAFFONT (1979), DE WIT et VAN WINDEN (1991), JOVANOVIC (1994).

La situation d'entrepreneur définie par ces modèles ne relève pas de l'analyse kirznerienne mais est plus proche d'une situation où un individu exploite des talents entrepreneuriaux déployables dans une activité indépendante (self-employment). Cela étant, les modèles proposés restent utiles 
dès lors que, comme nous le proposons, l'activité entrepreneuriale au sens de KIRZNER a été associée, en référence à un même individu, à d'autres activités productives pour cause d’incomplétude du système de marchés.

La probabilité d'être entrepreneur est une fonction croissante de l'écart entre le profit attendu à la suite de l'acte d'entreprendre et le revenu attendu d'une occupation comme salarié. Cet écart peut lui-même être déterminé par une série de facteurs. C’est ainsi que le facteur régional peut affecter la probabilité de création d'entreprise en participant, à côté d'autres facteurs tels que les capacités de l'individu, à la détermination du profit attendu (AUDRETSCH et FELDMAN, 2003). KANGASHARJU (2000) suggère en outre que le fait régional détermine le salaire. Il ajoute encore que la probabilité de création d'entreprise est aussi une fonction croissante de la richesse de l'individu ou du ménage. Celle-ci entre en effet dans la détermination de la capacité financière du candidat entrepreneur et est donc susceptible de relâcher la contrainte de financement ou de réduire la barrière à l'entrée formée par les coûts fixes d'installation et de production. En cela, il suit l'argument développé par EVANS et JOVANOVIC (1989) et lui donne une dimension régionale en ajoutant que la richesse des individus n'est pas identiquement distribuée d'une région à une autre.

Le fait régional importe dans l'explication du choix occupationnel. Cela étant, quels sont les traits caractéristiques de la région qui participent de ce point de vue, de manière significative, à la distinction des territoires?

Dans un contexte de dépression économique par exemple, l’individu considérera la probabilité de trouver un emploi salarié ou d'être au chômage. Il y trouvera peut-être une raison supplémentaire de devenir entrepreneur (STOREY, 1994). Dans le modèle qu'ils développent en référence aux travaux de Frank KNIGHT, KIHLSTROM et LAFFONT (1979) donnent aux individus le choix entre diriger une activité " hasardeuse » en créant leur propre entreprise et travailler pour un salaire non risqué. Leur modèle intègre l'aversion pour le risque comme facteur explicatif. A côté de ce facteur, les auteurs reconnaissent toutefois bien d'autres éléments qui viennent aussi influencer la décision quant au choix proposé : la capacité entrepreneuriale, l'habileté au travail, la capacité d'accès au capital requis pour créer la firme.

L'ensemble des arguments explicatifs précités (espérance de revenus en termes relatifs, aversion pour le risque) peuvent aisément être redimensionnés de manière à trouver une traduction tenant compte du contexte régional. Une région concentrant des industries en déclin devrait offrir relativement moins de perspectives de développement de l'emploi salarié. La situation pourrait inciter alors la population locale à se tourner davantage vers l'expérience entrepreneuriale. Cependant, un contexte économique déprimé au niveau local pourrait aussi agir négativement sur les intentions entrepreneuriales dès lors que les candidats entrepreneurs devant éventuellement servir la demande locale anticiperaient la faiblesse de cette dernière.

En référence au Sud italien, FLORIO (1996) montre comment la localisation d'entreprises de grande taille peut entamer le potentiel entrepreneurial des régions. Ayant donné suite à une politique de subvention publique particulièrement importante pour encourager l'installation de grosses unités de production industrielle dans le Mezzogiorno, quelques grandes entreprises ont agi de manière significative sur les choix occupationnels des individus en offrant des salaires très 
attractifs à la force de travail locale, affectant en conséquence les possibilités d’un développement endogène reposant sur la création d’activités indépendantes.

\subsection{La dimension régionale de la croissance économique entrepreneuriale}

Les moteurs de la croissance entrepreneuriale présentés par KIRZNER et ses épigones peuvent être articulés autour de trois arguments principaux :

- l'action entrepreneuriale d'arbitrage des prix accroît l'efficience économique et technologique. La réduction du gaspillage économique associé à la situation d’inefficience est un gain de croissance ;

- le progrès technologique permet de nouvelles opportunités de profit. En les découvrant, l'entrepreneur participe à l'accroissement de la productivité des facteurs de production ;

- l'action entrepreneuriale n'épuise pas seulement un stock d'opportunités de profit, éventuellement alimenté par la dépense délibérée de Recherche et Développement. En découvrant les opportunités de profit, l'entrepreneur peut en créer de nouvelles qui demeureront ignorées tant qu’une action entrepreneuriale ne les découvre à leur tour.

L'accroissement d'efficience découlant de l'action entrepreneuriale peut avoir deux types de conséquences selon le contexte spécifié. En s'attachant au cas statique le plus simple, où le stock d'opportunités de profit est tenu constant pour une économie régionale autarcique, l'action entrepreneuriale kirznerienne épuise une situation d'ignorance jusqu'à amener l'économie à sa situation d'équilibre. Dans ce cas, les sources entrepreneuriales de la croissance régionale apparaissent limitées. Une économie autarcique ayant atteint une situation de parfaite efficience technique et économique voit la disparition de ses entrepreneurs. En situation d'économie régionale ouverte et selon la situation relative de la région par rapport au reste du monde, les accroissements d'efficience de l'économie régionale peuvent être à l'origine d'avantages compétitifs. Cette idée trouve un soutien en dehors de l'approche néo-autrichienne de la croissance. A cet égard, notons par exemple la contribution de PORTER (1990). La valeur et l'originalité de l'approche kirznerienne demeurent toutefois d'identifier précisément la fonction spécifique de l'entrepreneur dans le processus de marché et de lui attribuer les améliorations d'efficience. En économie ouverte, la fonction équilibrante de l'entrepreneuriat doit être appréciée en rapport à la situation d'ignorance de l'économie dans son ensemble, économie de référence et reste du monde.

L’économie est l'objet de multiples changements. Parmi ces changements, certains résultent de l'accroissement ou de l'amélioration délibérés des facteurs de production. De nouveaux biens et services sortis de laboratoire de Recherche et Développement étendent chaque jour les possibilités de production et de satisfaction des consommateurs. Cela étant, KIRZNER et ses épigones s'accordent pour reconnaître les sources non entrepreneuriales de la croissance en ce compris, sans doute, les facteurs de croissance sur lesquels insistent les nouvelles théories de la croissance (R\&D, capital humain, capital technologique, capital physique, privé et public). Le changement induit par la croissance nourrit le stock d'opportunités de profit à découvrir par l'action entrepreneuriale. L'action entrepreneuriale est elle-même à l'origine de nouvelles opportunités (HOLCOMBE, 1998, 2003).

L'introduction de la dimension régionale dans la discussion des sources de croissance conduit d'abord à un constat selon lequel la répartition de la croissance et des facteurs de croissance n'est 
pas uniforme dans l'espace. Ensuite, elle pose une double question : y a-t-il des avantages en termes de croissance qui puissent directement être rapportés à l'organisation spatiale des ressources et des activités? Quelles sont plus particulièrement les implications de cette organisation spatiale sur le stock d’opportunités de profit et les découvertes entrepreneuriales ?

Ces questions ont été abordées, depuis une perspective néo-autrichienne plus ou moins marquée, par HOLCOMBE $(1998,2003)$ et par DESROCHERS $(1998,2001)$. L'explication des avantages liés à la concentration spatiale des activités peut certes aller bien au-delà des avantages à mettre en rapport direct avec l'action entrepreneuriale ${ }^{8}$. Nous nous limitons cependant à une présentation de ces derniers avantages.

Ainsi, pour ce qui concerne les avantages liés à la concentration spatiale des facteurs de production déployables et de la production en association avec l'action entrepreneuriale, notons :

- l'effet favorable attendu de la proximité géographique des actifs disponibles dans l'économie sur la fréquence des découvertes d’opportunité de profit. La concentration faciliterait la (re)combinaison des actifs dans de nouvelles applications productives ;

- les résultats d'activités innovantes ne sont pas aisément communicables à autrui. La difficulté tient à la nature même de l'innovation qui relève tout au moins partiellement d'une information tacite, par opposition à une information codifiée. Dans ce cas, la proximité entre l'entrepreneur et les tenants de l'activité innovante, en facilitant l'échange d'information tacite, agit favorablement sur la découverte des opportunités de profit liées aux résultats de l'activité d'innovation ;

- le caractère tacite de la vigilance entrepreneuriale elle-même a déjà été relevé précédemment (cf. section I, point 1). L'argument était invoqué pour expliquer la prise en charge par un même individu de plusieurs fonctions économiques en dehors de la fonction entrepreneuriale. Cependant, les difficultés de communication d'une information tacite étant atténuées par la proximité des agents concernés, la réalisation effective de l'opportunité de profit par l'entremise de gestionnaires chargés d'exécution devrait aussi en être facilitée. Le travail du gestionnaire devrait également être plus efficace et moins coûteux si celui-ci peut s'adosser localement à un vaste marché et à une capacité productive compétitive et diversifiée.

HOLCOMBE (2003, p. 36) souligne encore la concentration dans l'espace de l'entrepreneuriat et prend pour exemple la Silicon Valley. Si l'entrepreneuriat épuisait seulement les opportunités de profit, pareille concentration ne trouverait pas d'explication. Ce sont les entrepreneurs qui en l'occurrence découvrent de nouvelles opportunités de profit fondées sur les opportunités du passé. Parce que la vigilance entrepreneuriale se réfère à une information tacite et parce qu'elle est source d’opportunités de profit, elle suscite localement les découvertes entrepreneuriales.

L'entrepreneuriat transforme son environnement et le rend plus fécond en opportunités de profit comme chez HOLCOMBE. Dans leur étude de cas relative au développement de la Capitol Region aux Etats-Unis et à l'émergence d'un cluster industriel, FELDMAN (2001) et FELDMAN et FRANCIS (2002) ont montré que le fait régional détermine effectivement la création et le développement d'activités mais, avant toutes choses, ces auteurs mettent remarquablement en évidence le rôle essentiel qu'ont pu jouer les entrepreneurs eux-mêmes dans la création de ressources territoriales spécifiques jusqu'à ce que les phénomènes entrent dans des rapports de renforcement mutuel. 


\section{LES CONDITIONS DE DYNAMIQUES ENTREPRENEURIALES ET TERRITORIALES CUMULATIVES}

La section précédente a mis en évidence l'importance du contexte régional sur le choix occupationnel des individus, d'une part, et la croissance économique d'origine entrepreneuriale, d'autre part. La prise en compte du contexte régional rend nécessaire l'évaluation des effets en retour de la croissance et du développement d'activités sur les choix occupationnels. Dans cette troisième et dernière section, nous nous proposons d'examiner et de discuter à partir d'un schéma simplifié (cf. Schéma 1, ci-dessous) les résultats d’une approche systémique rendant compte des interactions entre les éléments précités ${ }^{9}$.

On pose préalablement l'hypothèse d'incomplétude du système de marchés rendant nécessaire la responsabilité multifonctionnelle de l'entrepreneur et celle d'autarcie (absence d'échanges commerciaux et de mouvements pendulaires ou migratoires).

Schéma 1 : Choix occupationnels, croissance et effets en retour

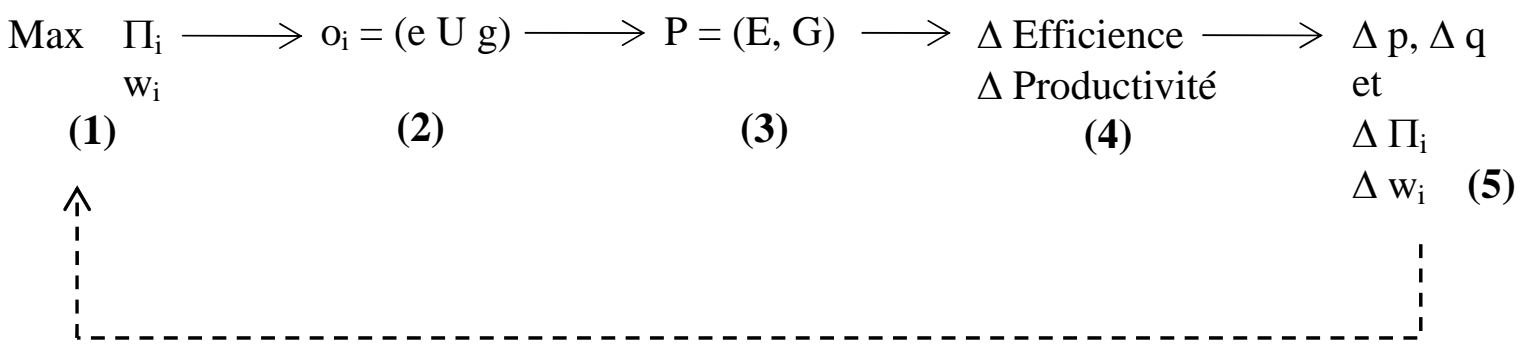

(6)

Soit une population P composée d’individus ayant le choix de leur occupation professionnelle : être entrepreneur ${ }^{10}$ ou gestionnaire. (1) Le choix de l'individu i est déterminé par le maximum des espérances de revenu qu'il recevrait de son emploi dans l'une ou l'autre activité, compte tenu de ses qualités personnelles et celles de son projet entrepreneurial. L'individu peut devenir entrepreneur [e] et s'attendre à la perception d'un profit $\Pi_{\mathrm{i}}$, ou devenir gestionnaire [g] contre un salaire attendu $\mathrm{w}_{\mathrm{i}}$. (2) Les choix individuels initiaux permettent alors la description de la population P selon un partage (3) entre l'entrepreneuriat [E], d'une part, et la gestion des activités [G], d'autre part. (4) L’économie se met en mouvement. Le partage initial de la population selon le choix occupationnel des individus détermine l'évolution de l'efficience technico-économique et des productivités factorielles, (5) rendant nécessaire la révision de tous les prix et quantités, et les revenus. Sans autre spécification, l'impact de l'entrepreneuriat sur les profits et les salaires est indéterminé. Notons néanmoins que ce sont ces nouveaux revenus qui détermineront les choix individuels futurs (6). Une condition pour qu'augmente, lors d'un second tour, la proportion d'entrepreneurs dans la population est d'obtenir en résultat des revenus entrepreneuriaux (profits attendus) qui soient supérieurs aux revenus attendus de gestionnaire. 
La théorie kirznerienne de la découverte entrepreneuriale suggère que la profitabilité de l'action entrepreneuriale est déterminée par l'intensité du déséquilibre de marché ou de l'ignorance étant donné les plans d'achat et de vente en présence dans l'économie. Aussi, c'est le marché, en produisant les prix et en dévoilant la profitabilité effective de l'action entrepreneuriale, qui permet de juger de la qualité de l'action entrepreneuriale. Dès lors que l'on incorpore le choix occupationnel des individus, c'est toute l'information, y compris l'information en provenance du marché du facteur de gestion qui doit être considérée.

Pour la discussion du cas d'une économie régionale, l'hypothèse d'autarcie apparaît forte. Les régions sont généralement plus perméables aux mouvements pendulaires et migratoires. La variation de la population active régionale complexifie l'anticipation des résultats attendus de l'action entrepreneuriale sur les choix occupationnels.

\section{CONCLUSIONS}

Dans cet article, nous nous proposions d'introduire la dimension régionale dans la théorie de la découverte entrepreneuriale de KIRZNER et de ses épigones. Deux aspects de cette théorie ont plus particulièrement retenu notre attention : d'abord, la notion de vigilance entrepreneuriale et l'absence de coût entrepreneurial d'un point de vue analytique ; ensuite, les effets de l'action entrepreneuriale sur la croissance. Nous avons suggéré que l'incomplétude du système de marchés pouvait justifier la responsabilité multifonctionnelle de l'entrepreneur. La réalisation effective de l'opportunité de profit par l'entrepreneur devenait conditionnelle à l'investissement de son temps et de ses capacités productives. De ce fait, l'acte entrepreneurial apparaissait aussi comme la composante d'un choix occupationnel, être entrepreneur et se trouver dans la nécessité d'assumer une série de fonctions extra-entrepreneuriales pour réaliser le profit ou être gestionnaire. L'introduction de la dimension régionale dans la problématique du choix occupationnel nous a notamment permis de souligner l'importance du contexte économique agrégé sur les paramètres du choix. Toutes les régions ne connaissent pas la même prospérité. Des résultats agrégés dépend l'allocation de la population active entre des activités entrepreneuriales ou managériales. Pour ce qui concerne la croissance, le caractère tacite de l'information liée à la vigilance entrepreneuriale ou aux activités innovantes nous a conduit à souligner particulièrement l'importance de rapports de proximité géographique entre les différents acteurs concernés. Enfin, une présentation systémique des arguments du choix occupationnel et de la croissance a conduit à mettre en évidence le fait que les effets de l'action entrepreneuriale sur la croissance pouvaient difficilement être considérés indépendamment des effets en retour sur les paramètres de choix individuels, $a$ fortiori en référence à un ensemble de ressources et d'activités économiques localisées en interrelation ou en interaction. 


\section{BIBLIOGRAPHIE}

AUDRETSCH, D.B., FELDMAN M.P. (2003), «Knowledge spillovers and the geography of innovation », prepared for HENDERSON, J.V., THISSE, J.F. (eds), Handbook of Regional and Urban Economics, Amsterdam, North-Holland, à paraître.

BALDWIN, R., FORSLID, R., MARTIN, P., OTTAVIANO, G., ROBERT-NICOUD, F. (2003), Economic geography and public policy, Princeton, New Jersey, Princeton University Press.

BECKER, G.S. (1965), «A theory of the allocation of time », Economic Journal, 75, pp. 493517.

BOETTKE, P.J., COYNE C.J. (2003), « Entrepreneurship and development: cause or consequence ", in R. KOPPL, ed., Austrian economics and entrepreneurial studies, Oxford, JAI - Elsevier Science, pp. 67-87.

CATIN, M. (1994), «Economies d'agglomération », in AURAY, J.-P., BAILLY, A., DERYCKE, P.-H., HURIOT, J.-M., eds, Encyclopédie d'économie spatiale - Concepts, Comportements, Organisations, Paris, Economica, pp. 107-109.

DEJARDIN, M. (2000a), « Entrepreneurship and economic growth: an obvious conjunction? An introductive survey to specific topics », Institute for Development Strategies DP, 8, Indiana University, Bloomington.

DEJARDIN, M. (2000b), "A model of entrepreneurial economic growth", Uddevalla Symposium 2000: Entrepreneurship, Firm Growth and Regional Development in the New Economic Geography, Trollhättan, June 15-17.

DEJARDIN, M. (2004), « La création d'entreprises et ses rapports au territoire. Retours critiques sur quelques résultats », mimeo.

DEMSETZ, H. (1983), « The neglect of the entrepreneur », in J. Ronen, ed., Entrepreneurship, Lexington, Massachusetts, LexingtonBooks, , pp. 271-280.

DESROCHERS, P. (1998), "A geographical perspective on Austrian economics », Quaterly Journal of Austrian Economics, 1, 2, pp. 63-83.

DESROCHERS, P. (2001), « Geographical proximity and the transmission of tacit knowledge », Review of Austrian Economics, 14, 1, pp. 25-46.

EVANS, D.S, JOVANOVIC, B. (1989), «An estimated model of entrepreneurial choice under liquidity constraints », Journal of Political Economy, 97, pp. 808-827.

FELDMAN, M.P. (2001), "The entrepreneurial event revisited : firm formation in a regional context », Industrial and Corporate Change, 10, 4, pp. 861-891. 
FELDMAN, M.P., FRANCIS, J. (2002), «Entrepreneurs and the formation of industrial clusters », mimeo.

FLORIO, M. (1996), « Large firms, entrepreneurship and regional development policy: 'growth poles' in the Mezzogiorno over 40 years », Entrepreneurship and Regional Development, 8, pp. 263-295.

FUJITA, M., THISSE, J.-F. (1997), « Economie géographique, problèmes anciens et nouvelles perspectives », Annales d'Economie et de Statistique, n²45, pp. 37-83.

FUJITA, M., KRUGMAN, P., VENABLES, A.J., (1999), The spatial economy. Cities, regions, and international trade, Cambridge, Massachusetts, The MIT Press.

GIFFORD, S. (1998), The allocation of limited entrepreneurial attention, Dordrecht, Kluwer Academic Publishers.

HARPER, D. A. (2003), Foundations of entrepreneurship and economic development, London, Routledge.

HEBERT, R.F., LINK, A. (1982), The entrepreneur. Mainstream views and radical critiques, New York, Praeger.

HOLCOMBE, R.G. (1998), «Entrepreneurship and economic growth », Quarterly Journal of Austrian Economics, 1, pp. 45-62.

HOLCOMBE, R.G. (2003), « The origins of entrepreneurial opportunities », Review of Austrian Economics, 16, pp. 25-43.

JOVANOVIC, B. (1994), « Firm formation with heterogeneous management and labor skills », Small Business Economics, 6, 3, pp. 185-193.

KANBUR, S.M. (1979), "Of risk taking and the personal distribution of income », Journal of Political Economy, 87, 4, pp. 719-748.

KANGASHARJU, A. (2000), « Regional variations in firm formation : panel and cross-section data evidence from Finland », Papers in Regional Science, 79, pp. 355-373.

KIHLSTRÖM, R.E., LAFFONT, J.J. (1979), « A General Equilibrium Entrepreneurial Theory of Firm Formation Based on Risk Aversion », Journal of Political Economy, 87, 4, pp. 719-748.

KIRZNER, I.M. (1973), Competition and entrepreneurship, Chicago, University of Chicago Press.

KIRZNER, I.M. (1979), « Alertness, luck, and entrepreneurial profit », paper presented in 1978 and reproduced in Perception, opportunity, and profit. Studies in the theory of entrepreneurship, Chicago, University of Chicago Press, pp. 154-181 et pp. 258-259. 
KIRZNER, I.M. (1982), "The theory of entrepreneurship in economic growth », in : C.A. Kent e.a., eds, Entrepreneurship, Englewood Cliffs, New Jersey, Ptrentice Hall, pp. 272-277.

KIRZNER, I.M. (1985), Discovery and the capitalist process, Chicago, University of Chicago Press.

KIRZNER, I.M. (1997), «Entrepreneurial discovery and the competitive market process : an Austrian approach », Journal of Economic Literature, XXXV, March, pp. 60-85.

KIRZNER, I.M. (1999), "Creativity and/or alertness : a reconsideration of the Schumpeterian entrepreneur », Review of Austrian Economics, 11, pp. 5-17.

KIRZNER, I.M. (2000), The driving force of the market : essays in Austrian economics, London, Routledge.

KNIGHT, F. (1921), Risk, uncertainty and profit, Boston, Houghton Mifflin.

KRUGMAN, P. (1991), «Increasing Returns and Economic Geography », Journal of Political Economy, Vol. 99, n³, pp. 483-499.

LUCAS, R.E. (1978), « On the size distribution of business firms », Bell Journal of Economics, 9, pp. 508-523.

PORTER, M.E. (1990), The competitive advantage of Nations, Londres, Macmillan.

ROMER, P. (1986), «Increasing returns and long run growth », Journal of Political Economy, 94(5), pp. 1002-1037.

ROMER, P. (1990), «Endogenous technical change », Journal of Political Economy, 98(5), pp. S71-S102.

SCHUMPETER, J.A. (1911), Theorie der wirtschaftlichen Entwicklung. Eine Untersuchung über Unternehmergewinn, Kapital, Kredit, Zins und den Konjunkturzyklus ; translated by R. Opie, The Theory of Economic Development. An Inquiry into Profits, Capital, Credit, Interest, and the Business Cycle, Oxford: Oxford University Press (1963 (1934)).

STOREY, D. J. (1994), Understanding the Small Business Sector, Londres, Routledge.

WIT, G. DE, VAN WINDEN, F. (1991), «An M-sector, N-group behavioral model of selfemployment », Small Business Economics, 3, 1, pp. 49-66. 
${ }^{1}$ Sur ces questions, voir en particulier HARPER (2003).

${ }^{2}$ L'existence d'un processus de marché équilibrant ou l'apprentissage du marché sont des arguments développés par KIRZNER et ses épigones qui suscitent un débat très nourri parmi les économistes néo-autrichiens, de sorte qu'il serait abusif de considérer ces contributions théoriques comme faisant partie définitive d'une connaissance commune à ces économistes. Pour une brève présentation des principales critiques émises à l'encontre de la théorie de la découverte entrepreneuriale, le lecteur intéressé pourra consulter avantageusement KIRZNER, 1997, section V, pp. 78-80.

${ }^{3}$ Cf. en particulier KIRZNER, 1985, pp. 21-25.

${ }^{4}$ Voir aussi GIFFORD (1998) qui modélise l'allocation de l'attention entrepreneuriale entre différentes activités dont le nombre n'est pas fixé, comme chez Becker, mais qui est déterminé par l'allocation de l'attention entrepreneuriale elle-même (GIFFORD, 1998, p. 2).

${ }^{5}$ « Sans le guide de marché que constituent le profit et les prix, la question globale de l'innovation doit être reconsidérée très sérieusement. De nouvelles méthodes de production peuvent ne pas augmenter nécessairement l'efficience ; de nouveaux produits peuvent être de nouveaux produits que personne ne veut. » (KIRZNER, 1982, p. 275 ; notre traduction).

${ }^{6}$ On peut encore se référer à cet auteur, en particulier HOLCOMBE (2003), pour une présentation des sources de la croissance économique qui ne relèvent pas de l'action entrepreneuriale kirznerienne mais de l'action mécanique.

${ }^{7}$ La dimension régionale est ici discutée lorsqu'elle peut être mise en rapport direct avec le choix occupationnel des individus. Pour une présentation plus étendue des effets liés à la dimension régionale sur la création d'activités économiques notamment à partir d'arguments tirés de l'économie industrielle, cf. DEJARDIN (2004).

8 Cf. l'abondante littérature développée autour des économies d'agglomération. Le concept d'économies d'agglomération n'est certes pas neuf en économie (voir CATIN, 1994). Ces économies sont toutefois particulièrement sous les feux de l'actualité scientifique depuis l'essor de la Nouvelle Economie Géographique (KRUGMAN, 1991) et depuis que se multiplient dans ce domaine de recherche les efforts de modélisation sous une forme rigoureuse (voir notamment FUJITA et THISSE, 1997 ; FUJITA, KRUGMAN et VENABLES, 1999 ; BALDWIN, FORSLID, MARTIN, OTTAVIANO et ROBERT-NICOUD, 2003).

${ }^{9}$ La résolution par voie de simulation d'un modèle comparable au modèle schématisé est proposée par DEJARDIN (2000b).

${ }^{10}$ La proposition suppose que chaque individu soit porteur d'une découverte entrepreneuriale. 